\title{
Calcifying Odontogenic Cyst - A Case Report and Literature Review
}

\author{
Elitsa Deliverska
}

Department of Oral and Maxillofacial surgery, Faculty of Dental Medicine, Medical University, Sofia

\begin{abstract}
Background: The calcifying odontogenic cyst (COC) is a rare example of a developmental odontogenic cyst. COC may clinically be diagnosed as calcifying epithelial odontogenic tumour, adenomatoid odontogenic tumour, ameloblastic fibroodontoma, complex or compound odontoma, dentigerous cyst or as other types of odontogenic cysts.

Purpose: The aim is to present an unusual clinical case of COC associated with an impacted third molar of the lower jaw.

Material and methods: We present a male patient with histologically proved COC associated with a retained lower left third molar. The orthopantomography revealed an impacted third molar whose crown was surrounded by a radiolucent, circular in shape amorphous formation.

Results: The treatment of choice was third molar surgery - odontectomia and extirpation/enucleation of the cyst formation. The complete removal of the cyst was successfully performed, since after few years of follow-up there were no signs of recurrence. The result of the pathophysiological examination showed COC.

Conclusion: $\mathrm{COC}$ is a rare odontogenic cyst of developmental origin, which should be diagnosed timely and surgically removed.
\end{abstract}

Keywords: COC, Ghost cell, third molar

\section{Background}

A calcifying odontogenic cyst (COC) is rarely found in everyday practice in oral surgery. It was thoroughly described for the first time by Gorlin (1) and co-workers in 1962 who were impressed by the significant presence of the so-called "ghost cells." At that time, they suggested that this cyst may represent the oral counterpart of the dermal calcifying epithelioma of Malherbe (2, 3). In 1963 Gold introduced the term 
keratinizing and calcifying odontogenic cyst. He believed that this specific type of cyst had not been described previously in the literature as an entity (4).

Over the years, since its first description, it has become clear that the calcifying ghost cell odontogenic cyst (CGCOC) has a number of variants, including features of a benign odontogenic tumour. It was classified as SNOMED code 930/0, in the World Health Organization's (WHO) publication Histological Typing of Odontogenic Tumours $(5,6,7)$. The World Health Organization's classification (WHO), according to Kramer and Pindborg, from 1992 (4) and the majority of the authors favoured the use of the term calcifying odontogenic cyst and described it as a cystic or neoplastic-like odontogenic pathological lesion of the jaw and classified it as a benign odontogenic tumour (7).

There has been a complete re-evaluation of this lesion by many authors. One major conclusion of Praetorius et al. about this lesion was that it comprised two entities: a cyst and a neoplasm (8).

The calcifying ghost cell odontogenic cyst (CGCOC) or COC is a rare example of a developmental odontogenic cyst, its occurrence constituting about $0.37 \%$ to $2.1 \%$ of all odontogenic pathological changes of the jaws $(9,10,11)$, although it can be found together with other odontogenic tumours, most frequently with odontoma in $24 \%$ of the cases (11). It most often occurs as a central (intraosseous) lesion $(11,12,13)$, whereas peripheral (extraosseous) localization in the soft tissue is rare (14) Their incidence (of all types summarily) is $13-25 \%(10,15,16,17)$. In $65 \%$ of cases a calcifying odontogenic cyst occurs in the anterior part of the jaw in the incisor and canine region $(5,6,11,16)$. Central and peripheral forms of the calcifying odontogenic cyst occur equally in the upper and lower jaw $(8,10,11)$. Johnson (17) reported the occurrence of $60 \%$ in the mandible, $30 \%$ in the form of peripheral calcifying odontogenic cysts, while the anterior part of the jaw was involved in $53 \%$ of cases. On the basis of 52 examples of calcifying odontogenic cysts connected with an odontoma, Hirshberg et al. (18) concluded that the upper jaw was affected in $61.5 \%$ and the anterior region of the jaw in $75 \%$.

COC can occur in very young patients, even in the first year of life. Cases have also been recorded of patients in their eighties $(6,9,10,18)$. However, in the majority of cases it occurs in the second decade of life $(5,6,8,12,16)$.

COC may be clinically diagnosed as a calcifying epithelial odontogenic tumour, an adenomatoid odontogenic tumour, an ameloblastic fibroodontoma, complex or compound odontoma, a dentigerous cyst or as other types of odontogenic cysts. It is unclear whether it should be regarded as a separate entity or as a stage in the development of another odontogenic tumour. $(15,16,17,18,19,20,21,22)$

The reasons why in the past we did not have the diagnosis of COC are probably associated with the clinical and histological similarity of the lesion with some odontogenic tumors or cysts. The lesion differs histologically from odontogenic cysts and epithelial tumours such as ameloblastoma, but could be similar to the calcifying epithelial odontogenic tumour that presents with more aggressive growth. The presentation of the following case, diagnosed two years ago, with no recurrence until now, could be our contribution for the better understanding of this lesion in our clinical practice. 


\section{Case}

A 46-year-old man was referred to the Department of Oral Surgery with a severe pain of the left side of the mandible. No visible facial asymmetry could be seen, but a pain was detected by intraoral palpation in the vestibulum behind the second left molar of the lower jaw. Also intraorally a sharply circumscribed swelling could be seen on the left side of the mandible $1 \times 1,5 \mathrm{~cm}$. The swelling was covered with unchanged mucosa. No paresthesia of the lower lip was present.

The radiographic findings disclosed a unilocular well-circumscribed round in shape radiolucency around the crown of an impacted third molar. The distal root of the left second molar was not resorbed (Figure 1).
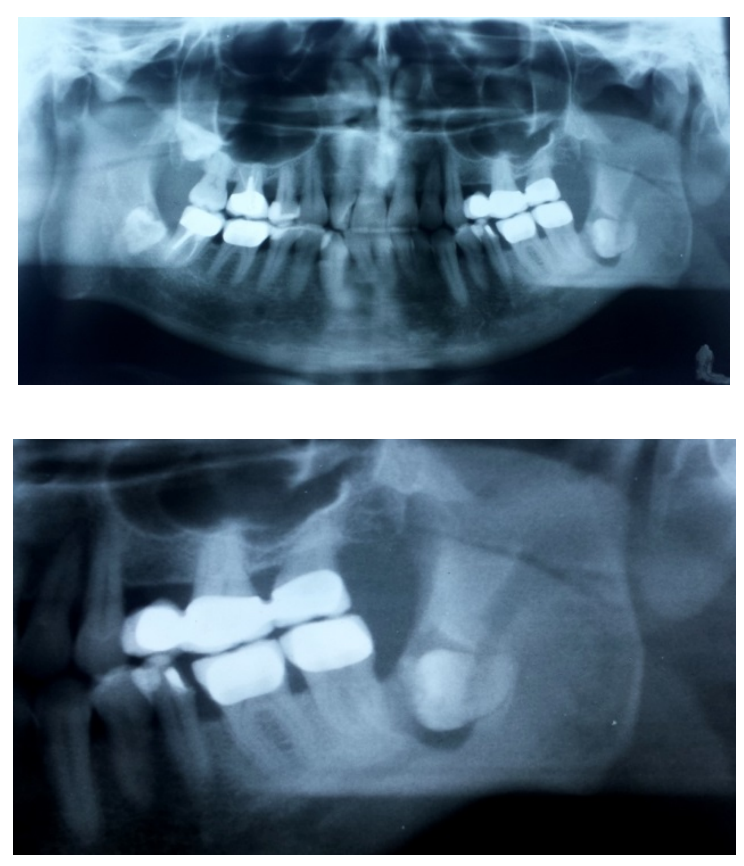

Fig. 1 Orthopantomography

The differential clinical diagnosis included: Follicular cyst, odontogenic keratocyst, odontogenic myxoma, ameloblastic fibroodontoma, monocystic ameloblastoma and other rare odontogenic tumours.

Because of the size of the cyst the operation was performed under local anaesthesia. The third molar was removed and the cystic lesion was enucleated in accordance with the principle of clinical methods for treating small cystic lesions of jaws.

Histologically the wall was composed of fibrous tissue, and the internal surface covered in some places by a thin layer or in others by a thick layer of epithelial cells (Specimen No 140443/04.2014). There were hilic, and in places calcified areas, and ghost cells. Tiny islets of odontogenic epithelia could be seen in the connective stroma (Figure 2). 


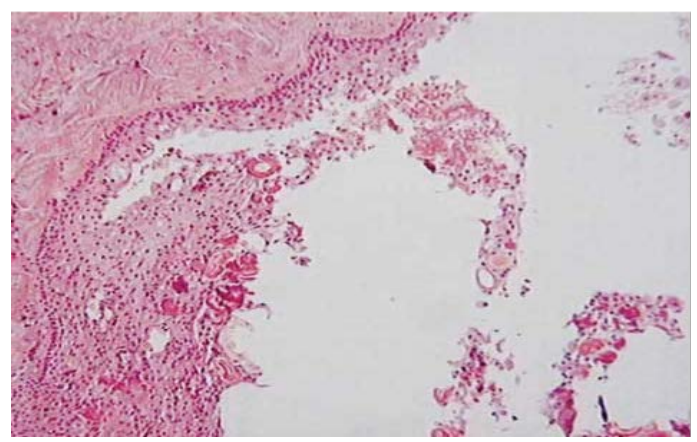

Fig.2 Histological findings

\section{Discussion and Literature Review}

We have described a rare case of calcifying an odontogenic cyst associated with an impacted third molar treated in the Department of Oral and Maxillofacial Surgery, Medical University, Sofia.

$\mathrm{COC}$ is a rear lesion of the jaws and was first described by Gorlin and colleagues. They identified file CGCOC as a distinct pathological entity in 1962, although according to Altini and Farman, the condition had previously been described in the German literature in 1932 by Rywkind (9). It was earlier thought to be an oral presentation of the dermal calcifying epithelioma of Malherbe $(6,10)$.

Table1: Commonly used terminologies for CGCOC $(6,23)$

\begin{tabular}{|c|c|}
\hline Terminology & Author proposed \\
\hline Calcifying odontogenic cyst (COC) & Gorlin et al. (1962) \\
\hline $\begin{array}{l}\text { Keratinizing Calcifying odontogenic } \\
\text { cyst }(\mathrm{KCOC})\end{array}$ & Gold et al. (1963) \\
\hline Keratinizing ameloblastoma (KA) & Bhaskar (1965) \\
\hline $\begin{array}{l}\text { Calcifying ghost cell odontogenic } \\
\text { tumor (CGOT) }\end{array}$ & Fejerskov and Krogh (1972) \\
\hline $\begin{array}{l}\text { Cystic calcifying odontogenic tumor } \\
\text { (CCOT) }\end{array}$ & Freedman et al. (1975) \\
\hline $\begin{array}{l}\text { Dentinogenic ghost cell tumor } \\
\text { (DGCT) }\end{array}$ & Praetorius et al. (1981) \\
\hline $\begin{array}{l}\text { Epithelial odontogenic ghost cell } \\
\text { tumor (EOGCT) }\end{array}$ & Ellis and Shmooker (1986) \\
\hline $\begin{array}{l}\text { Calcifying ghost cell odontogenic } \\
\text { cyst (CGCOC) }\end{array}$ & Toida (1998) \\
\hline $\begin{array}{l}\text { Odontogenic ghost cell tumor } \\
\text { (OGCT) }\end{array}$ & Colmenero et al. (1990) \\
\hline $\begin{array}{l}\text { Odontogenic ghost cell } \\
\text { ameloblastoma (OGCA) }\end{array}$ & Shear (1994) \\
\hline $\begin{array}{l}\text { Odontocalcifying Odontogenic } \\
\text { tumor (OOT) }\end{array}$ & Wirshberg et al. (1994) \\
\hline $\begin{array}{l}\text { Calcifying cystic odontogenic tumor } \\
\text { (CCOT) }\end{array}$ & WHO classification (2005) \\
\hline
\end{tabular}

Controversy and confusion exist regarding the relationship between non-neoplastic, cystic lesions and solid tumour masses that share the cellular and histomorphologic features described by authors $(9,11,16$, 19). In 1971, the WHO described CGOC as a "non-neoplastic" cystic lesion; nevertheless, it decided that the lesion should be classified as a benign odontogenic tumour. In 1992, the World Health Organization (WHO) classified CGOC as a neoplasm rather than a cyst but confirmed most of the cases are non- 
neoplastic. In view of this duality, many different terminologies have been applied to cystic and solid CGOC variants, but calcifying odontogenic cyst is the preferred term $(19,23)$. Different terminologies for CGOC are reviewed in Table 1. Several classifications of CGOC subtypes have been proposed, but most of them have limitations in separating cystic and neoplastic variants (2).

The first classification is proposed by Praetorius et al. (8).

Type 1. Cystic type:
A - simple unicystic type,
B - odontoma-producing type,
C - ameloblastomatous proliferating type.

Type 2. Neoplastic type: dentinogenic ghost cell tumour.

A recent classification of CGOC has been suggested by Reichart (11).

1 Non-neoplastic (simple cystic) variant (CGCOC):
a. -with non-proliferative epithelial lining;
b. -with non-proliferative (or proliferative) epithelial lining associated with odontomas;
c. -with proliferative epithelial lining;
d. -with unicystic, plexiform ameloblastomatous proliferation of epithelial lining.

2 Neoplastic variant:

A. benign type (CGCOT):

i. cystic subtype (cystic CGCOT)

ii. solid subtype (solid CGCOT)

B. malignant type (malignant CGCOT or OGCC):

iii. cystic subtype,

iv. solid subtype.

$3 \quad$ Calcifying ghost cell odontogenic cyst.

$4 \quad$ Also classified as compound (or complex) cystic ghost cell odontomas.

5 Does not completely fulfill the histopathologic criteria of early ameloblastoma as suggested by Vickers and Gorlin.

$6 \quad$ Calcifying ghost cell odontogenic tumour.

$7 \quad$ With histopathologic features of early ameloblastoma as suggested by Vickers and Gorlin.

8 Resembling a peripheral amelobastoma, hence termed peripheral epithelial odontogenic ghost cell tumour.

9 Often called central epithelial odontogenic ghost cell tumour. Odontogenic ghost cell carcinoma. 
CGCOC is a rare developmental cyst. Tomich (5) reviewed about 34 years for odontogenic tumors and cysts at Indiana University School of Dentistry, and he found that only 51 cases of calcifying ghost cell odontogenic cysts were diagnosed-less than two cases per year! It follows that the average oral and maxillofacial surgeon is likely to see only a case or two during his/her professional career (5). In 1992 Bucher (11) described a multi-cystic form of calcifying odontogenic cyst as a separate entity on the basis of an analysis of 215 clinical and histological features of central calcified odontogenic cysts. Instead of calcifying odontogenic cyst Langlais and co-workers suggested the term calcifying odontogenic lesion including cystic and tumorous form as separate forms, and a combined lesion when elements of both forms are observed.

The odontogenic origin of the CGCOC is widely accepted $(10,13,21,22)$. The cells responsible for the calcifying odontogenic cyst are dental lamina rests (rests of Serres) within either the soft tissue or bone. Therefore, calcifying ghost cell odontogenic cysts are cysts of primordial origin and are not associated with the crown of an impacted tooth (22). It most often occurs as a central (intraosseous) lesion (11), whereas peripheral (extraosseous) localization in the soft tissue is rare (14).

There is an almost even gender distribution. In Asians, it shows a higher incidence in younger age groups; almost $70 \%$ occur in the second and third decades, whereas in whites, only about $53 \%$ occur in the respective decades. Moreover, in Asians, the lesions show a predilection for the maxilla (65\%), whereas in whites, the predilection is for the mandible $(62 \%)(21,22)$. The most common site of occurrence has been the anterior part of the jaws. In the mandible, several cases have crossed the midline, but this is less usual in the maxilla (22). In our case report, the age of the male patient was 46 years and the condition occurred at the posterior region of the mandible, which is not a classical feature for this lesion.

The central CGCOC (intraosseous) presents as an asymptomatic hard swelling of the jaw that produces expansion rather than erosion of the bone. Pain usually indicates a secondary infection (10). The cysts are usually discovered as an incidental radiographic finding. Early in their development, they will appear completely radiolucent. As they mature, they develop calcifications that produce a well-circumscribed, mixed radiolucent-radiopaque appearance. Three general patterns of radiopacity are seen. One is a saltand-pepper pattern of flecks, the second is a fluffy cloud-like pattern throughout, and the third is a crescent-shaped pattern on one side of the radiolucency in a "new moon"-like configuration $(10,15)$. Calcifying odontogenic cyst is most frequently radiographically seen as a unilocular translucency with sharply circumscribed edges occurring in the form of a multilocular lesion in a very small number of cases, from $5 \%$ to13\%. $(10,15)$

In our case report, radiographic examination disclosed a solitary well-circumscribed round radiolucency with single calcified material within it. A definite diagnosis of calcifying odontogenic cyst can be reliably made on the basis of a histological examination due to the lesion's lack of characteristic clinical and radiological features, as well as its variable biological behavior.

The histological features of a classic calcifying ghost cell odontogenic cyst are characteristic. The microscopical features of the lesion shows a fibrous capsule with a lining of odontogenic epithelium. The basal layer is made up of ameloblast-like columnar or cuboidal cells of 4-10 cell thickness. It is covered by loosely arranged epithelial cells bearing similarity to the stellate reticulum of the enamel organ (Figure 2). There are varying numbers of epithelial cells which are devoid of nuclei, eosinophilic, and retaining their 
basic cell outline (ghost cells). These ghost cells may undergo calcification and lose their cellular outline to form a sheet like-area. Many investigators have made efforts to clarify the nature of ghost cells by employing special histochemical methods, transmission electron microscopy, and scanning electron microscopy, and various theories have been proposed without any general agreement. (4) Gorlin et al., Ebling and Wagner, Gold, Bhasker, Komiya et al. and Regezi et al. (1-10), all believed that ghost cells represent normal or abnormal keratinization. Levy suggested that they represent squamous metaplasia with subsequent calcification caused by ischemia. Sedano and Pindborg (7) thought the ghost cells represented different stages of normal and aberrant keratin formation and that they were derived from the metaplastic transformation of odontogenic epithelium. Other investigators suggested or implied that ghost cells may represent the product of an abortive enamel matrix in odontogenic epithelium. However, the morphology of ghost cells seems different from that of an enamel matrix $(21,22,23)$. Ghost cells are not unique to CGCOC, but are also seen in odontoma, ameloblastoma, craniopharyngioma, and other odontogenic tumours $(2,6)$. The treatment of cystic lesions involves enucleation with a long-term follow-up period. Recurrence depends on the completeness of the cyst's removal. Prognosis is good for cystic CGCOC and less certain for neoplastic $\operatorname{CGCOC}(21,25,26)$. The differential diagnosis of CGCOC could be made with odontogenic cyst: follicular cyst, odontogenic keretocyst, odontogenic tumours such as adenomatoid odontogenic tumour, ameloblastic fibro-odontoma ameloblastic fibroma, and ameloblastoma where the treatment and prognosis in such cases is based on the aggressiveness of the lesion.

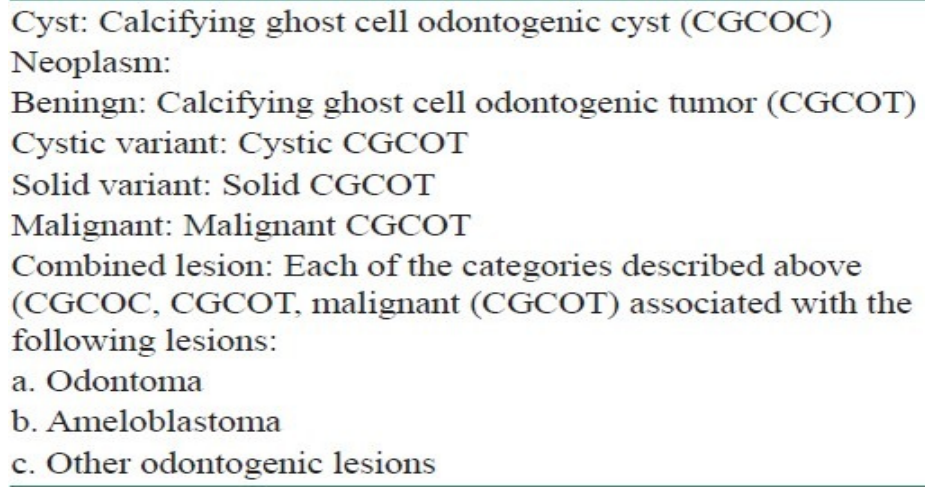

Table 2 Classification proposed by Toida (1998)

With regard to the very small number of recurrences, only eight cases of recurrences have been documented in the English literature.

The treatment of calcifying odontogenic cyst is usually enucleation of the cyst. In his most recent publication on pathology Barnes mentions the possibility of a malignant transformation, and in differential diagnosis points out that $\mathrm{COC}$ must be differentiated from calcifying odontogenic tumours and ameloblastoma, which are essentially more aggressive and require an extensive surgical approach.(26) The diagnosis of pathological alterations of odontogenic etiology appears very simple, however, only to those with a superficial knowledge of the matter. The further one considers the problem of odontogenic tumours, the more profound knowledge of the structure is necessary for correct diagnosis, both on the part of the clinician and the pathologist, who need to have subspecialist training in this field. An optimal solution would be the existence of a clinical pathologist who would be present at the final diagnosis in cooperation with an oral or maxillofacial surgeon. 


\section{Conclusion}

Our case represents the classical features of calcifying odontogenic cyst, according to Praetorius et al. It comes under category of Type 1(a) simple unicystic type, and according to Reichart's classification, it comes under the category of calcifying ghost cell odontogenic cyst (CGCOC) non-neoplastic variant. COC may mimic numerous odontogenic or non-odontogenic lesions. Therefore its clinical and histological diagnosis is difficult. This case demonstrates, in addition, that specific knowledge in oral histopathology is required to differentiate odontogenic lesions.

\section{References}

1. Gorlin RG, Pindborg JJ, Clausen FP, Vickers RA. The calcifying odonlogcnic cyst-a possible analogue of the cutaneous calcifying epithelioma of Malherbe: an analysis of fifteen cases. Oral Surgery, Oral Medicine, Oral Pathology. 1962;15(10):1235-1243.

2. Shafer WG, Hine MK, Levy BM. A Textbook of Oral Pathology Cyst and Tumours of Odontogenic Origin. 4th edition. Orlando, Fla, USA: W. b. Saunders; 2001.

3. Shear M, Speight P. Cyst of the Oral and Maxillofacial Region. 4th edition. New York, NY, USA: Blackwell Munksgaard; 2007. Calcifying odontogenic cyst (Calcifying cystic odontogenic tumors) pp. 100-107.

4. Gold L. The keratinizing and calcifying odontogenic cyst. Oral Surgery, Oral Medicine, Oral Pathology. 1963;16(12):1414-1424.

5. Tomich CE. Calcifying odontogenic cyst and dentinogenic ghost cell tumour. Oral and Maxillofacial Surgery Clinics of North America. 2004;16(3):391-397

6. Thinakaran $M$, Sivakumar $P$, Ramalingam S, Jeddy N, Balaguhan S. Calcifying ghost cell odontogenic cyst: A review on terminologies and classifications, J Oral Maxillofac Pathol. 2012 Sep-Dec; 16(3): 450-453.

7.KRAMER I.R. PINDBORG HJJSHEAR M. Histological typung of odontogenic tumours. Springer Verlag, Berlin 1992.

8. Praetorius F, Hjorting-Hansen E, Gorlin RJ, Vickers RA. Calcifying odontogenic cyst. Range, variations and neoplastic potential. Acta Odontologica Scandinavica. 1981;39(4):227-240.

9. Altini VIS, Farman AG. The calcifying odontogenic cyst. eight new cases and a review of the literature. Oral Surgery Oral Medicine and Oral Pathology. 1975;40(6):751-759.

10. Erasmus JH, Thompson IOC, Van Rensburg LJ, Van der Westhuijzen AJ. Central calcifying odontogenic cyst. A review of the literature and the role of advanced imaging techniques. Dentomaxillofacial Radiology. 1998;27(1):30-35. 
11. Buchner A. The central (intraosseous) calcifying odontogenic cyst: an analysis of 215 cases. Journal of Oral and Maxillofacial Surgery. 1991;49(4):330-339.

12. Cawson RA. Essentials of Oral Pathology and Oral Medicine. 6th edition. Orlando, Fla, USA: W.B. Saunders; 1991.

13. Shear M. Developmental odontogenic cysts-an update. Journal of Oral Pathology and Medicine. 1994;23(1):1-11. 11.

14. Swan, R.H., Houston, G.D., Moore, S.P. Peripheral calcifying odontogenic cyst (Gorlin Cyst), J Periodontol. 1985;56:340-343.

15. lida S, Fukuda Y, Ueda T, Aikawa T, Arizpe JE, Okura M. Calcifying odontogenic cyst : radiological findings in 11 cases. Oral Surgery, Oral Medicine, Oral Pathology, Oral Radiology, and Endodontology. 2006;101(3):356-362.

16. Philpsen HP, Reichart PA. Odontogenic Tumours and Allied Lesions. 1st edition. Hanover Park, III, USA: Quientessence Publishing; 2004.

17. Johnson A, Fletcher M, Gold L, Chen SY. Oral Maxillofac. J Oral Maxillofac Surg. 1997 Jul;55(7):679-83.

18. Hirshberg A, Kaplan I, Buchner A. Calcifying odontogenic cyst associated with odontoma: A possible separate entity (odontocalcifying odontogenic cyst). J Oral Maxillofac Surg 1994; 52: 555-8.

19. Hong SP, Ellis GL, Hartman KS. Calcifying odontogenic cyst. A review of ninety-two cases with reevaluation of their nature as cysts or neoplasms, the nature of ghost cells, and subclassification. Oral Surgery Oral Medicine and Oral Pathology. 1991;72(1):56-64.

20. Marx RE, Stern D. Oral and Maxillofacial Pathology: A Rationale for Diagnosis and Treatment. 1st edition. Hanover Park, III, USA: Quientessence Publishing; 2003. Odontogenic and nonodontogenic cysts; p. 607.

21. Neville BW, Damm DD, Allen CM, Bouquot JE. Oral and Maxillofacial Pathology. 2nd edition. Philadelphia, Pa, USA: W. B. Saunders; 2002. Odontogenic cysts and tumours; p. 590.

22. Kenzevic G, Sokler K, Kobler P, Manojlovic S. Calcifying odontogenic cyst-Gorlin's cystreport of two cases coll. Antropol. 2004;28(1):357-362.

23. Toida M. So-called calcifying odontogenic cyst: review and discussion on the terminology and classification. Journal of Oral Pathology and Medicine.1998;27(2):49- 52.

21. Rushton VE, Horner K. Calcifying odontogenic cyst-a characteristic. British Journal of Oral and Maxillofacial Surgery. 1997;35(3):196-198.

22. Chen SY, Miller AS. Ultrastructure of the keratinizing and calcifying odontogenic cyst. Oral Surgery, Oral Medicine, Oral Pathology. 1975;39(5):769-780. 
23. Sonone A, Sabane VS, Desai R. Calcifying Ghost Cell Odontogenic Cyst: Report of a Case and Review of LiteratureCase Rep Dent. 2011;2011:328743.

24. Saghafi S, Zare-Mahmoodabadi R, Salehinejad J, Kadeh $H$, Afzal-Aghaee $M$. Immunohistochemical analysis of p53 and PCNA expression in calcifying odontogenic cyst. J Oral Sci. 2010;52:609-13

25. Kler S, Palaskar S, Shetty VP, Bhushan A. Intraosseous calcifying odontogenic tumour. J Oral maxillofac Pathol. 2009;13:27-9.

26. de Fatima Bernandes V, de Lacerda JC, de Aguiar MC, Gomez RS. Calcifying odontogenic cyst associated with an orthokeratinized odontogenic cyst. Head Neck

\section{Corresponding author:}

Corresponding author: Sofia 1431

Str."Georgi Sofiiski"1

Faculty of Dental medicine

Medical University Sofia

Department of Oral and Maxillofacial surgery

Associate prof. Elitsa Deliverska, PhD 